\title{
Interaction between Pseudomonas aeruginosa and Staphylococcus aureus: description of an anti- staphylococcal substance
}

\author{
Z. A. MACHAN, T. L. PITT*, W. WHITE, D. WATSONt, G. W. TAYLORt, P. J. COLE and R. WILSON‡ \\ Host Defence Unit, Department of Thoracic Medicine, National Heart and Lung Institute, Emmanuel Kaye Building, \\ Manresa Road, London SW3 6LR, "Division of Hospital Infection, Central Public Health Laboratory, 61 Colindale \\ Avenue, London NW9 5HT and t Department of Clinical Pharmacology, Royal Postgraduate Medical School, Du Cane \\ Road, London W12 ONN
}

\begin{abstract}
Summary. The presence of Pseudomonas aeruginosa in the sputum of 191 patients with cystic fibrosis was significantly related $(\mathrm{p}<0.0001)$ to the absence of Staphylococcus aureus. Crossstreaking tests showed that 40 of 50 clinical strains of $P$. aeruginosa produced substances that inhibited the growth of $S$. aureus. When incorporated into agar plates, this antibacterial substance(s) inhibited the growth of 177 of 189 strains of nine staphylococcal species, all of 16 methicillin-resistant $S$. aureus and 27 of 39 strains of six other gram-positive genera. The substance(s) did not inhibit 23 strains of seven gram-negative genera tested. The antibacterial activity was heat stable and could be extracted into chloroform; activity was retained on Sephadex G-15 $\left(\mathrm{V} / \mathrm{Vo} \approx 2, \mathrm{M}_{\mathrm{r}}<500\right)$ and eluted as a single peak from high performance liquid chromatography, well separated from pseudomonic acid, pyocyanin and a number of other phenazines.
\end{abstract}

\section{Introduction}

Staphylococcus aureus is usually the initial bacterial pathogen in the lungs of children with respiratory disease accompanying cystic fibrosis (CF); with increasing age, Pseudomonas aeruginosa begins to predominate. ${ }^{1}$ Although antibiotic therapy is probably a major factor contributing to the decline of the staphylococci, bacterial antagonism may also play an important part in the establishment of $P$. aeruginosa in the lungs of these patients. The ability of $P$. aeruginosa to antagonise other bacteria was first demonstrated in 1899. ${ }^{2,3}$ Forty-two years later, Schoental ${ }^{4}$ prepared three antibacterial extracts of $P$. aeruginosa : the phenazine pigments, pyocyanin and 1-hydroxyphenazine, and an almost colourless substance derived from an old culture. Young ${ }^{5}$ confirmed the activity of these extracts but found that the antibiotic effect was absent from non-pigmented cultures. The report that biologically prepared 1hydroxyphenazine has antibiotic activity ${ }^{4}$ was probably due to contamination by pyocyanin. ${ }^{6-8}$ Some studies have shown that $P$. aeruginosa produces substances antagonistic towards $S$. aureus. The mechanism of inhibition of staphylococcal growth may be due to nutrient depletion in mixed growth with

Received 26 Jan. 1990; revised version accepted 31 Aug. 1990. * Correspondence should be sent to Dr R. Wilson, Host Defence Unit, Department of Thoracic Medicine, National Heart and Lung Institute, Emmanuel Kaye Building, Manresa Road, London SW3 6LR.
P. aeruginosa, the production of specific metabolic inhibitors, or tolytic enzyme(s). ${ }^{9-11}$ We have identified a low-molecular-weight hydrophobic antibacterial factor, distinct from pyocyanin and other phenazines, which is produced by clinical isolates of $P$. aeruginosa and which may be responsible for the anti-staphylococcal activity of $P$. aeruginosa in CF patients.

\section{Materials and methods}

\section{Bacteria}

Fifty clinical isolates of $P$. aeruginosa, mostly from CF patients, and 323 isolates of other species tested for sensitivity to the antibacterial activity of $P$. aeruginosa were obtained from cultures submitted to the Division of Hospital Infection, Central Public Health Laboratory, for epidemiological typing. Grampositive bacteria included 261 staphylococci: $S$. aureus (99 strains, 16 of which were methicillin resistant), S. capitis (15) S. epidermidis (33), S. haemolyticus (19), $S$. hominis (16), S. saprophyticus (29), S. simulans (26), $S$. warneri (14), S. xylosus (10); and 39 strains of six other genera, Micrococcus spp. (5 strains), Corynebacterium spp. (6), Bacillus spp. (14), Listeria spp. (8), Kurthia zopfii (2), and faecal streptococci (4). Gramnegative bacteria tested included: Acinetobacter spp. (3 strains), Enterobacter cloacae (3), Escherichia coli (3), Haemophilus influenzae (4), Klebsiella pneumoniae (3), Proteus spp. (4) and Serratia marcescens (3). Other 
Pseudomonas spp. tested were also obtained from DHI and included: $P$. fluorescens (4 strains), $P$. putida (2), $P$. cepacia (2), $P$. mendocina (2) and $P$. diminuta (2).

Bacteria were grown on nutrient agar (Oxoid) and, when appropriate, on horse blood $(7 \% \mathrm{v} / \mathrm{v})$ agar or chocolate agar. The following media were used in addition : Kings " $A$ " agar, ${ }^{12}$ mannitol salt agar (MSA; Oxoid), Pseudomonas Isolation Agar (PIA; Difco), Cystine-Lactose Electrolyte Deficient Agar and Broth (CLED; Oxoid), Tryptone Soy Broth (TSB; Oxoid), and Todd-Hewitt Broth (Difco). Plates were incubated at $37^{\circ} \mathrm{C}$ for up to $48 \mathrm{~h}$. Culture filtrates were prepared from broth cultures of $P$. aeruginosa which were centrifuged at $8000 \mathrm{~g}$ for $30 \mathrm{~min}$ and the supernates were filtered through a $0 \cdot 45-\mu \mathrm{m}$ filter (Gelman Sciences Ltd, Northampton). Sputum obtained from 191 patients (aged 16 years or more) with $\mathrm{CF}$, was digested by pancreatin (BDH, Poole, Dorset) at $37^{\circ} \mathrm{C}$ for $2 \mathrm{~h}^{13}$ and plated on selective and non-selective agar for the isolation of $P$. aeruginosa and $S$. aureus.

\section{Antibacterial activity}

Antibacterial activity produced by different strains of $P$. aeruginosa was demonstrated in four ways.

Cross-streak tests. An agar plate was seeded with a 4-h TSB culture of a strain of $\boldsymbol{P}$. aeruginosa applied with a sterile cotton swab in a $1-\mathrm{cm}$ wide streak. After incubation at $37^{\circ} \mathrm{C}$ for $24 \mathrm{~h}$, the bacterial growth was removed from the plate with a microscope slide and the residual micro-organisms were killed by exposure to chloroform vapour for $30 \mathrm{~min}$. TSB cultures of staphylococcal strains were diluted one in 100 in fresh broth and streaked with a loop at right angles to the line of the original inoculum. The plates were incubated at $37^{\circ} \mathrm{C}$ for $18 \mathrm{~h}$ and examined for inhibition of the indicator strain in the area that had supported the growth of the producer strain.

Well-plate assay. Lawns of the staphylococcal strain S1940 were prepared by flooding the surface of a Kings " $A$ " agar plate with a 3-h broth culture. The excess fluid was removed and the surface of the agar was allowed to dry in air. Wells cut in the agar with a sterile metal punch ( $7 \mathrm{~mm}$ diameter) were filled with $200 \mu \mathrm{l}$ of filtrates of $48-\mathrm{h}$ broth cultures of $P$. aeruginos $a$ strain P3940 or fractions obtained by high performance liquid chromatography (HPLC). The plate was incubated at $37^{\circ} \mathrm{C}$ for $18 \mathrm{~h}$ and the zone of inhibition of growth of the staphylococcus was recorded.

Growth in mixed culture. Four-hour CLED broth cultures of $P$. aeruginosa strain P3940 or S. aureus strain S1940 were diluted with fresh broth to give equal optical density at $600 \mathrm{~nm}$ as measured on a nephelometer $\left(\mathrm{OD}_{600}=0 \cdot 10\right)$, and were then mixed in equal $(5-\mathrm{ml})$ volumes. Controls of the same volume were prepared from sterile broth and also from broth cultures of each organism. During incubation at $37^{\circ} \mathrm{C}$, 100- $\mu \mathrm{l}$ samples were taken at 20-min intervals for $1 \mathrm{~h}$, hourly intervals for $6 \mathrm{~h}$, and again after $24 \mathrm{~h}$. Viable counts were determined by a standard dilution technique on MSA, PIA and Kings "A" agar media. To determine whether changes in viable count observed were due to pre-formed antibacterial factor, a filtrate $(5 \mathrm{ml})$ of a 4-h $P$. aeruginos a broth culture was added to a 4-h $S$. aureus broth culture $(5 \mathrm{ml})$. A chloroform extract of $10 \mathrm{Kings}$ " $\mathrm{A}$ " agar plates on which $P$. aeruginosa $\mathrm{P} 3940$ had been cultured for $24 \mathrm{~h}$ was added to a 4-h $S$. aureus broth culture. A chloroform extract of 10 Kings "A" agar plates was used as a control. Viable counts were performed at similar intervals, as above.

Agar-plate incorporation. Nutrient agar plates were prepared containing either partially-purified factor (from Sep Pak ${ }^{\circledR}$, see below), $20 \mu \mathrm{g} / \mathrm{ml}$ loaded in $200 \mu \mathrm{l}$ of methanol, or an equivalent volume of methanol vehicle as a control. A 36-rod applicator was used to apply $0.3 \mu \mathrm{l}$ of diluted broth cultures of 244 grampositive strains containing $c .10^{4} \mathrm{cfu} / \mathrm{ml}$ on to the plates which were then incubated at $37^{\circ} \mathrm{C}$. Growth wás recorded after overnight culture and after 1 week at room temperature.

\section{Purification and characterisation of antibacterial factor(s) from $P$. aeruginosa}

A non-pigmented clinical isolate of $P$. aeruginosa strain P3940 was grown on Kings " $A$ " agar plates at $37^{\circ} \mathrm{C}$ for $24 \mathrm{~h}$. The bacterial growth was removed and the agar was cut into small pieces, transferred to a conical flask and extracted with chloroform (three volumes of $500 \mathrm{ml}$ each per 100 plates). The chloroform extract was loaded on to 10 silica Sep-Paks ${ }^{\circledR}$ (Waters Associates, Harrow, Middlesex) and the activity was eluted with methanol. HPLC was performed with a Waters Associates dual pump system on a $\mu$ Bondapak $\mathrm{C}_{18}$ column $(30 \mathrm{~cm} \times 0.8 \mathrm{~cm})$, eluting at $2 \mathrm{ml} / \mathrm{min}$ with a 25-min linear gradient of propan-2-ol in aqueous acetic acid 5\% (20:80-40:60 v:v). ${ }^{14}$ Fractions ( $\left.2 \mathrm{ml}\right)$ were assayed by the well-plate technique after solvent removal under vacuum. The factor was also subjected to chromatography on Sephadex G-15 $(32 \times 1.5 \mathrm{~cm}$; Pharmacia, Milton Keynes) eluting at $30 \mathrm{ml} / \mathrm{h}$ in methanol:ammonia $(\cdot 880)$ : water $(2: 1: 2 \mathrm{v}: \mathrm{v}: \mathrm{v}) .^{15}$ Fractions $(3 \mathrm{ml})$ were collected. Samples of the antibacterial factor were treated with either $2 \mathrm{M} \mathrm{HCl}$ $\left(60^{\circ} \mathrm{C}, 18 \mathrm{~h}\right)$, acetic anhydride in methanol $(1: 3 \mathrm{v}: \mathrm{v}$, $\left.1 \mathrm{~h}, 20^{\circ} \mathrm{C}\right)$ or methanolic hydrogen chloride $(0.4 \mathrm{M}$, $\left.1 \mathrm{~h}, 20^{\circ} \mathrm{C}\right) .{ }^{14}$ All solvents and reagents were removed under vacuum and resuspended in phosphate-buffered saline (PBS) for assay by the well plate or mixed culture method. Reagents blanks were inactive in the assay. Authentic pyocyanin and 1-hydroxyphenazine standards were synthesised as previously described. ${ }^{6,8,16}$

\section{Results}

Samples of sputum from 191 patients with CF were examined and 184 were found to be infected with 
$P$. aeruginosa, S. aureus or both. P. aeruginosa was present in the sputum of 146 of 191 patients, of which $67(46 \%)$ were also colonised by $S$. aureus. Of the 45 patients who did not yield $P$.aeruginosa from their sputum, $38(84 \%)$ were positive for $S$.aureus. There was a positive correlation between the presence of $P$. aeruginosa and the absence of $S$. aureus $\left(\chi^{2}=176.89 ; \mathrm{p}<0.0001\right)$, suggesting that suppression of $S$. aureus growth by $P$. aeruginosa may have occurred.

In an initial screen, 40 of $50 P$. aeruginosa strains inhibited the growth of six randomly selected strains of $S$. aureus in the cross-streak assay and produced large $(\sim 30 \mathrm{~mm})$ zones of inhibition. Four of the $P$. aeruginosa strains were weakly active, and the remaining six did not inhibit the growth of any of the staphylococci tested. Five strains of $P$. aeruginosa that exhibited strong anti-staphylococcal activity were tested for activity against 50 randomly selected strains of $S$.aureus. The growth of all staphylococci was suppressed by each of the Pseudomonas strains but the zones of inhibition varied with the agar medium used. Inhibition of staphylococcal growth was maximal on CLED and Kings " $A$ " agar but was markedly reduced on blood agar. Further tests with other Pseudomonas spp. showed that anti-staphylococcal activity was particularly associated with $P$. aeruginosa: only two of four strains of $P$. fluorescens inhibited the growth of 10 strains of $S$. aureus (randomly selected from the original 50) whereas two strains each of P. putida, $P$. cepacia, $P$. mendocina and $P$. diminuta did not affect staphylococcal growth.

$P$. aeruginosa $\mathrm{P} 3940$, a non-pigmented strain with strong anti-staphylococcal activity, was incubated with sensitive strain $S$. aureus S1940 in broth culture. Within 20 min the viable count of the staphylococcus was reduced to below detectable levels, and that of the pseudomonas remained constant. Regrowth of strain S1940 did not occur after overnight incubation in the presence of $P$. aeruginosa (fig. 1). P. aeruginosa strain P3888, which did not inhibit the growth of $S$. aureus S1940 in preliminary cross-streak tests, did not reduce the viable count of strain S1940 after incubation in mixed culture for $6 \mathrm{~h}$ (fig. 1).

A 4-h culture filtrate of $P$. aeruginosa P3940 did not decrease the viability of $S$. aureus, whereas an 18-h culture filtrate from $P$. aeruginosa decreased the viability of $S$. aureus by one order of magnitude (c.10fold). The antibacterial activity present in the $18-\mathrm{h}$ culture filtrate was completely removed by chloroform extraction and a much greater effect ( 10000 -fold) was observed when a chloroform extract of agar (after an 18-h growth of $P$. aeruginosa $\mathrm{P} 3940$ was removed) was incubated with $S$. aureus S1940 (fig. 2). These results suggest that this strain produces a lipid-like factor or factors that inhibit the growth of $S$. aureus. After overnight incubation, regrowth of the staphylococcus was observed; this could have resulted from exhaustion or inactivation of the bactericidal activity, as subcultures of $S$. aureus $\mathrm{S} 1940$ taken from the vessel at $6 \mathrm{~h}$

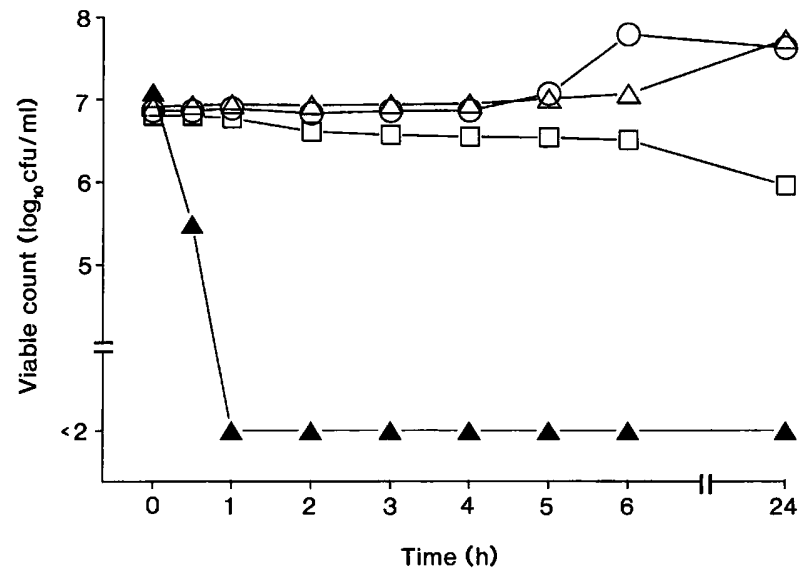

Fig. 1. The effect of $P$. aeruginosa on the growth of $S$. aureus in mixed culture. Viable counts (cfu/ml) are shown for $S$. aureus $\mathrm{S} 1940$ in mixed culture $(\boldsymbol{\Lambda}-\boldsymbol{\Lambda})$ with $\boldsymbol{P}$. aeruginosa $\mathrm{P} 3940(\mathrm{O}-\mathrm{O})$, with controls showing the growth of $S$. aureus $S 1940$ alone $(\triangle-\triangle)$ and during co-incubation with a second strain of $P$. aeruginosa (P3888) which was inactive in the cross-streak test $(\square-\square)$. The limit of detection of the assay was $10^{2} \mathrm{cfu} / \mathrm{ml}$.

remained sensitive to fresh extract of $P$. aeruginosa P3940.

The antibacterial activity was soluble in chloroform, but only poorly soluble in PBS or ether; it was not destroyed by boiling $(30 \mathrm{~min})$, freezing or mild acid hydrolysis, although a partial reduction in activity did occur after acetylation and esterification, suggesting the presence of amino, hydroxyl or carboxyl functions. Activity eluted as a single low molecular weight peak on Sephadex G15 (V/Vo $\approx 2,<500 \mathrm{Da})$; elution was undertaken with methanolic ammonia, a solvent system originally developed for the gel filtration of leukotriene $\mathrm{D}_{4}{ }^{15}$ to overcome poor water solubility.

To obtain sufficient purified material for further study, P. aeruginosa $\mathrm{P} 3940$ was grown on $100 \mathrm{Kings}$ "A" agar plates and activity was extracted with chloroform. Activity was retained on silica Sep-Paks ${ }^{\circledR}$ and could be eluted with methanol. Small amounts of the factor were purified further by reverse phase HPLC. The major peak of bioactivity eluted in fractions $33 / 34$, well separated from pseudomonic acid (fraction 23/24) and the common pseudomonas phenazines-pyocyanin, 1-hydroxyphenazine, 1-carboxyphenazine and phenazine-1-carboxyamide (fractions 9-14, fig. 3). The high percentage of organic modifier in the elution solvent indicated the hydrophobic nature of the material.

Using the Sep-Pak ${ }^{\circledR}$-purified material, the activity of the antibacterial factor was tested by the agar plate assay against 244 gram-positive bacteria (table). At a concentration of $20 \mu \mathrm{g} / \mathrm{ml}$, this material inhibited the growth, during incubation for $24 \mathrm{~h}$, of 193 of 205 strains of staphylococci, including the 16 methicillinresistant $S$. aureus (MRSA) strains and a high-level pseudomonic acid-resistant strain of $S$. aureus (MIC $>1028 \mathrm{mg} / \mathrm{L}$ ). When the plates were kept at room temperature for a further 7 days and re-examined: 57 


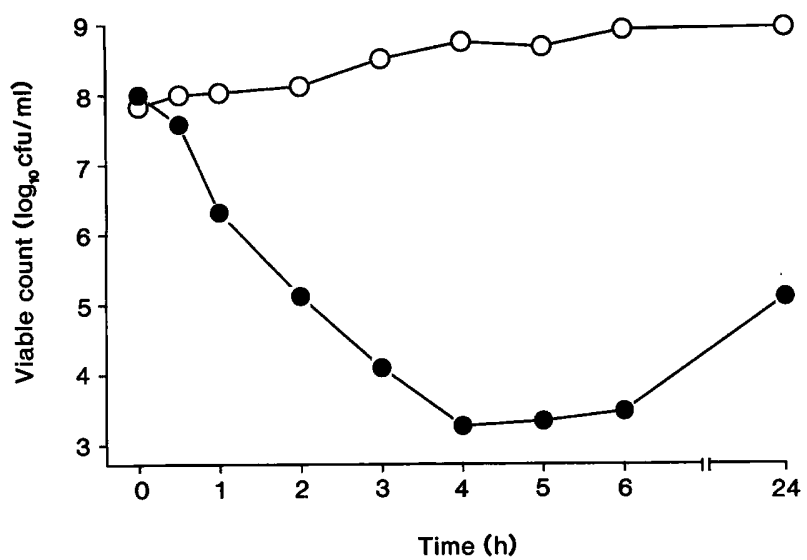

Fig. 2. A chloroform extract of $10 \mathrm{Kings} \mathrm{"A"} \mathrm{agar} \mathrm{plates} \mathrm{on} \mathrm{which}$ $P$. aeruginosa $\mathrm{P} 3940$ had been cultured for $24 \mathrm{~h}$ caused a rapid reduction in the viability of $S$. aureus $\mathrm{S} 1940$ ( - - ), which showed some recovery after $24 \mathrm{~h}$. A chloroform extract of 10 non-inoculated Kings "A" agar plates alone had no effect $(\mathrm{O}-\mathrm{O})$. Viability of S. aureus $\mathrm{S} 1940$ is expressed as cfu/ml.
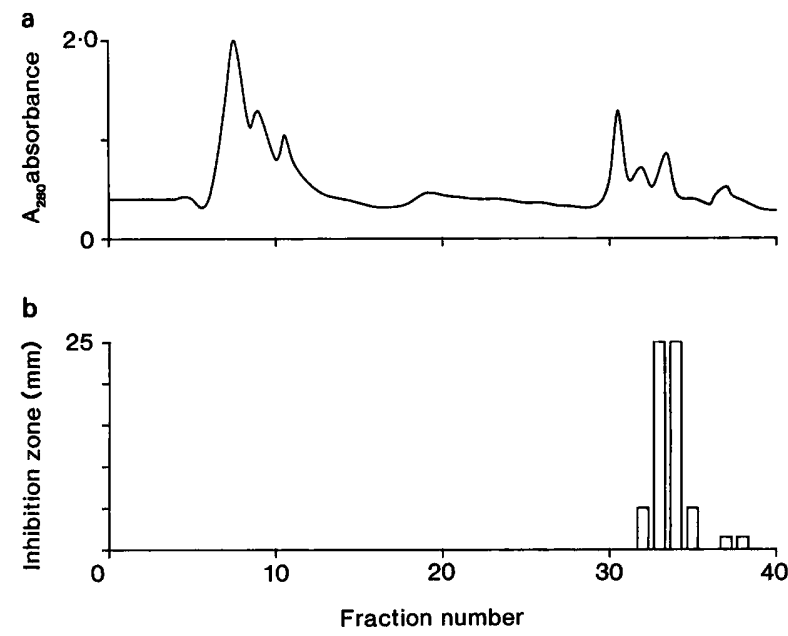

Fig. 3. HPLC profile of the antibacterial factor showing (a) $\mathbf{A}_{280}$ UV absorbance and (b) antibacterial activity ( $\square$ ). Activity is expressed as the size of the zone of inhibition in the well-plate assay. Activity elutes in fractions $33 / 34$, and is well resolved from pyocyanin and the phenazines (fractions 9-14) and pseudomonic acid (fraction 23/24).

of 189 strains produced macroscopic growth. Furthermore, 27 of 39 strains of the six other gram-positive genera tested were inhibited by the factor after incubation for $24 \mathrm{~h}$ : Micrococcus spp. (5 of 5), Corynebacterium spp. (6 of 6), Bacillus spp. (12 of 14), Listeria spp. (2 of 8), Kurt. zopfii (2 of 2) and faecal streptococci (0 of 4).

However, the antibacterial factor was inactive against 23 strains from seven gram-negative genera tested. In comparison, pyocyanin $32 \mu \mathrm{g} / \mathrm{ml}$ had a wider spectrum of activity, inhibiting the growth of 50 of 56 strains of $S$. aureus and 14 of the same group of 23 gram-negative strains: Acinetobacter spp. (3 of 3), Ent. cloacae (3 of 3), E. coli (2 of 3), H. influenzae (4 of 4), $K$. pneumoniae (2 of 3), Proteus spp. (0 of 4), and
Table. Effect of partially-purified pseudomonas antibacterial factor $(20 \mu \mathrm{g} / \mathrm{ml})$ in nutrient-agar plates on the growth of staphylococci

\begin{tabular}{lc|cc}
\hline & & \multicolumn{2}{|c}{$\begin{array}{c}\text { Number of strains } \\
\text { growing on agar plates at }\end{array}$} \\
\cline { 2 - 3 } Staphylococcus sp. & $\begin{array}{c}\text { Number } \\
\text { tested }\end{array}$ & $24 \mathrm{~h}$ & 7 days \\
\hline S. aureus & 27 & 0 & 0 \\
S. capitis & 15 & 0 & $6(2)$ \\
S. epidermidis & 33 & 0 & $6(4)$ \\
S. haemolyticus & 19 & $1(1)$ & $5(3)$ \\
S. hominis & 16 & $1(1)$ & $4(4)$ \\
S. saprophyticus & 29 & $5(5)$ & $20(11)$ \\
S. simulans & 26 & $1(1)$ & $3(2)$ \\
S. warneri & 14 & $4(4)$ & $7(6)$ \\
S. xylosus & 10 & 0 & 6 \\
MRSA & 16 & 0 & $\ldots$ \\
\multicolumn{1}{c}{ Total } & 205 & $12(12)$ & $57(32)$ \\
\hline
\end{tabular}

(n), Number of bacteria that exhibited poor growth on agar incorporating the factor.

S. marcescens (0 of 3). 1-Hydroxyphenazine $32 \mu \mathrm{g} / \mathrm{ml}$ was inactive against all strains tested.

\section{Discussion}

Colonisation of the respiratory tract by $P$. aeruginosa may be aided, in part, by the production of antibacterial factors active against the resident commensal flora and other pathogenic bacteria. The apparent reduction in staphylococcal colonisation of adult CF patients consequent to pseudomonas infection prompted us to investigate the production of an antibacterial factor expressed by $P$. aeruginosa.

There have been several previous reports identifying antimicrobial factors from $P$. aeruginosa $a^{5,17,18}$ but these substances cannot account for the activity of $P$. aeruginosa described here. Pseudomonic acid and the phenazines have a different spectrum of antibacterial activity and are readily separated from the antibacterial factor by HPLC. The substance reported by Zyskind et al. ${ }^{19}$ which inhibited $S$. aureus was of high molecular weight. The action of the factor described in the present paper is rapid on S.aureus, both in mixed culture and when added to broth, in contrast to that found by Perestelo et al. ${ }^{20}$ who reported an anti-staphylococcal effect of $\boldsymbol{P}$. aeruginos $a$ only after incubation for $16 \mathrm{~h}$. The microcins originally described as a new family of antibiotics produced by $E$. coli ${ }^{21}$ are now known to be distinct chemical classes of antibiotics, with markedly variable structure and molecular weight. ${ }^{22,23}$ In the initial report, ${ }^{21}$ evidence was adduced that two strains of $P$. aeruginosa also produced microcins with antibacterial activity, although both had some activity against $E$. coli and are thus distinct from the factor reported in our study.

The anti-staphylococcal activity produced by 
$P$. aeruginosa may explain the high correlation between the absence of $S$. aureus in the presence of $P$. aeruginosa in CF, and the association of $P$. aeruginosa colonisation in CF patients with a decline in staphylococcal infections. We have purified this antibacterial factor, and shown that it is a low molecular weight hydrophobic species. Because of these properties, it may escape neutralisation by the host defence system in vivo and be a further factor contributing to the success of pseudomonas colonisa-

\section{References}

1. Mearns MB. Natural history of pulmonary infection in cystic fibrosis. In: Sturges JM (ed) Perspective in cystic fibrosis. Proceedings of the 8th International Cystic Fibrosis Conference. Toronto, Canadian Cystic Fibrosis Association. 1980: 325-334.

2. Bouchard $C$. Influence qu'exerce sur la maladie charbonneuse l'inoculation du bacille pyocyanique. C R Acad Sci 1989; 108: 713-714.

3. Emmerich R, Löw O. Bakteriolytische Enzyme als Ursache der erworbenen Immunität und die Heilung von Infectionskrankheiten durch dieselben. Z Hyg Infektkr 1899; 31 : 165.

4. Schoental $R$. The nature of the antibacterial agents present in Pseudomonas pyocyanea cultures. Br J Exp Pathol 1941; 22: 137-147.

5. Young G. Pigment production and antibiotic activity in cultures of Pseudomonas aeruginosa. J Bacteriol 1947; 54: 109-117.

6. Armstrong AV, Stewart-Tull DES, Roberts JS. Characterisation of the Pseudomonas aeruginosa factor that inhibits mouse-liver mitochondrial respiration. J Med Microbiol $1971 ; 4: 249-262$.

7. Watson D, MacDermot J, Wilson R, Cole PJ, Taylor GW. Purification and structural analysis of pyocyanin and 1hydroxyphenazine. Eur J Biochem 1986; 159: 309-313.

8. Wilson R, Pitt T, Taylor G et al. Pyocyanin and 1-hydroxyphenazine produced by Pseudomonas aeruginosa inhibit the beating of human respiratory cilia in vitro. $J$ Clin Invest 1987; 79 : 221-229.

9. Collins-Thompson DL, Aris B, Hurst A. Growth and enterotoxin B synthesis by Staphylococcus aureus S6 in associative growth with Pseudomonas aeruginosa. Can J Microbiol 1973; 19: 1197-1201.

10. Brito N, Falcón MA, Carnicero A, Gutiérrez-Navarro AM, Mansito TB. Purification and peptidase activity of a bacteriolytic extracellular enzyme from Pseudomonas aeruginosa. Res Microbiol 1989; 140: 125-137. tion and establishment of $P$. aeruginosa in the lungs of patients with $\mathrm{CF}$. The activity against a number of staphylococci, including MRSA strains and at least one high-level pseudomonic acid-resistant strain, raises the possibility that this substance may have some clinical application.

We thank Mr H. Todd (NHLI) and colleagues at the Central Public Health Laboratory (Colindale Avenue, London) for assistance in culturing and testing bacterial strains.

11. Troller JA, Frazier WC. Repression of Staphylococcus aureus by food bacteria. II. Causes of inhibition. Appl Microbiol $1963 ; 11: 163-165$.

12. King EO, Ward MK, Raney DE. Two simple media for the demonstration of pyocyanin and fluorescin. J Lab Clin Med 1954; 44 : 301-307.

13. Rawlins GA. Liquefaction of sputum for bacteriological examination. Lancet $1953 ; 2$ : 538-539.

14. Morris HR, Taylor GW, Piper PJ, Tippins JR. Structure of slow-reacting substance of anaphylaxis from guinea-pig lung. Nature 1980; 285: 104-106.

15. Morris HR, Taylor GW, Piper PJ, Sirios P, Tippins JR. Slowreacting substance of anaphylaxis: purification and characterisation. FEBS Lett 1978; 87: 203-206.

16. Wilson R, Sykes DA, Watson D, Rutman A, Taylor GW, Cole PJ. Measurement of Pseudomonas aeruginosa phenazine pigments in sputum and assessment of their contribution to sputum sol toxicity for respiratory epithelium. Infect Immun 1988; 56: 2515-2517.

17. Fuller AT, Banks GT, Mellows G, Barrow KD, Woolford M, Chain EB. Pseudomonic acid: an antibiotic produced by Pseudomonas fluorescens. Nature 1971; 234 : 416-417.

18. Hays EE, Wells IC, Katzman PA et al. Antibiotic substances produced by Pseudomonas aeruginosa. J Biol Chem 1945; 159: 725-750.

19. Zyskind JW, Pattee PA, Lache M. Staphylolytic substance from a species of pseudomonas. Science 1965; 147: 14581459.

20. Perestelo FR, Blanco M-T, Gutierrez-Navarro AM, Falcón MA. Growth inhibition of Staphylococcus aureus by a staphylolytic enzyme from Pseudomonas aeruginosa. Microbios Letters 1985 ; 30: 85-94.

21. Asensio C, Perez-Diaz JC, Martinez MC, Baquero F. A new family of low molecular weight antibiotics from enterobacteria. Biochem Biophys Res Commun 1976; 69: 7-14.

22. Kurepina NE, Khmel IA. Microcins: their nature and genetic determination. Mol. Gen Mikrobiol Virusol 1986; 4: 3-9.

23. Garcia-Bustos JF, Pezzi N, Asensio C. Microcin 7: purification and properties. Biochem Biophys Res Commun 1984; 119: 779-785. 
